\title{
Real-time photoacoustic flow cytography and photothermolysis of single circulating melanoma cells in vivo
}

\author{
Yun $\mathrm{He}^{1}$, Lidai Wang ${ }^{1, \dagger}$, Junhui Shi ${ }^{1,}$ Junjie Yao ${ }^{1}$, Lei Li ${ }^{1}$, Ruiying Zhang ${ }^{1}$, Chih-Hsien Huang ${ }^{2}$, \\ Jun Zou ${ }^{2}$, Lihong V. Wang ${ }^{1,3}$ \\ ${ }^{1}$ Department of Biomedical Engineering, Washington University in St. Louis, St. Louis, MO \\ 63130, USA. \\ ${ }^{\dagger}$ Current address: Department of Mechanical and Biomedical Engineering, City University of \\ Hong Kong, Kowloon, Hong Kong, China. \\ ${ }^{2}$ Department of Electrical and Computer Engineering, Texas A\&M University, College Station, \\ TX 77843, USA. \\ ${ }^{3}$ Current affiliation: Caltech Optical Imaging Laboratory, Department of Medical Engineering, \\ California Institute of Technology, Pasadena, CA 91125, USA
}

\begin{abstract}
Metastasis is responsible for as many as $90 \%$ of cancer-related deaths, and the deadliest skin cancer, melanoma, has a high propensity for metastasis. Since hematogenous spread of circulating tumor cells (CTCs) is cancer's main route of metastasis, detecting and destroying CTCs can impede metastasis and improve patients' prognoses. Extensive studies employing exogenous agents to detect tumor-specific biomarkers and guide therapeutics to CTCs have achieved promising results, but biosafety remains a critical concern. Taking another approach, physical detection and destruction of CTCs is a safer way to evaluate and reduce metastasis risks. Melanoma cells strongly express melanosomes, providing a striking absorption contrast with the blood background in the red to near-infrared spectrum. Exploiting this intrinsic optical absorption contrast of circulating melanoma cells, we coupled dual-wavelength photoacoustic flow cytography with a nanosecondpulsed laser killing mechanism that specifically targets melanoma CTCs. We have successfully achieved in vivo label-free imaging of rare single CTCs and CTC clusters in mice. Further, the photoacoustic signal from a CTC immediately hardware-triggers a lethal pinpoint laser irradiation that lyses it on the spot in a thermally confined manner. Our technology can facilitate early inhibition of metastasis by clearing circulating tumor cells from vasculature.
\end{abstract}

Keywords: Photoacoustic microscopy, flow cytography, circulating tumor cell, melanoma, laser therapy

\section{INTRODUCTION}

Melanoma is the most deadly type of skin cancer[1]. As with most types of cancer, up to $90 \%$ of its mortalities are attributed to metastases, mostly via hematogenous dissemination of circulating tumor cells (CTCs)[2]. Thus early detection and eradication of melanoma CTCs are vital for assessing and reducing the risks of metastasis and improving patients' prognoses[3, 4]. CTCs are very difficult to detect in the blood because there is only one CTC per million to billion red blood cells (RBCs). Clinical ex vivo CTC detection with high sensitivity heavily depends on labeling biomarkers on the cell membrane with exogenous agents, and the biosafety and labeling efficiency of these agents remain critical concerns, limiting their clinical translation[5].

Compared to other biological tissues, melanoma cells have high melanosome expression, providing a good endogenous absorption contrast with the blood background in the red to near-infrared (NIR) spectrum[6]. Hence, photoacoustic (PA) imaging, which provides the highest possible sensitivity to absorption contrast[7], is a good tool for label-free imaging of melanoma cells[8]. PA flow cytography based on a high-speed scanning mechanism can achieve both single-cell resolution and a fast imaging frame rate, making it ideal for detecting melanoma CTCs[9].

Photons Plus Ultrasound: Imaging and Sensing 2017, edited by Alexander A. Oraevsky, Lihong V. Wang, Proc. of SPIE Vol. 10064, 100641E · (c) 2017 SPIE · CCC code: 1605-7422/17/\$18 · doi: 10.1117/12.2255068 
Traditional clinical therapies for tumor metastasis remain palliative, with many therapeutics not reaching metastasis sites[10]. Taking another approach, therapies directly and physically targeting CTCs can reduce tumor metastasis risks with minimal side effects[11]. Physicians have long used nanosecond-pulsed lasers at 680-1064 nm wavelengths to treat melanocytic skin lesions with a high degree of selectivity and a low risk of postoperative complications[12]. By applying the same method on CTCs, radiant energy is largely confined in the absorbers, melanosomes, and can achieve selective photothermolysis of the absorbing cells[13].

Here, using our new dual-wavelength PA flow cytography, we report high-speed melanoma CTC imaging in combination with on-the-fly CTC photothermolysis at the single-cell level by pinpoint lethal irradiation from a nanosecond-pulsed therapy laser. We obtained high-resolution images of both single CTCs and CTC clusters in mice, and performed real-time CTC destruction in vivo.

\section{SYSTEM DESIGN}

Melanosome absorbs comparably to hemoglobin at $532 \mathrm{~nm}$, but $>10$ times more strongly than hemoglobin at 1064 $\mathrm{nm}[6]$. To exploit this absorption contrast against an RBC background, we developed a fast-scanning PA flow cytography system for high-speed imaging at both $532 \mathrm{~nm}$ and $1064 \mathrm{~nm}$ wavelengths. The system combines singlecell resolution imaging with on-the-fly selective CTC destruction by nanosecond-pulsed NIR laser-induced photothermolysis (Figure 1). The flow cytography employed a high-repetition-rate picosecond laser (APL-40001064, Attodyne Inc.; $500 \mathrm{kHz}$ maximum repetition rate) to provide simultaneous laser pulses at both $1064 \mathrm{~nm}$ and $532 \mathrm{~nm}$. The two laser pulses were combined through a longpass dichroic mirror and focused onto the imaging object. We employed a lab-made MEMS scanner resonating at $500 \mathrm{~Hz}$ to enable fast one-axis optical scanning over a long range. The motor stage holding the object provided mechanical scanning in the orthogonal direction. The excited acoustic waves were also reflected by this MEMS scanner, passed through the optical-acoustic splitter, and acquired by a $75 \mathrm{MHz}$ ultrasonic transducer. The whole system had a $\sim 7 \mathrm{um}$ resolution and a $10 \mathrm{~Hz}$ frame rate over a $3 \times 0.25 \mathrm{~mm}^{2}$ region. The frame rate was adjustable by tuning the scanning range and the laser repetition rate. The two laser pulses each excited an acoustic wave, and the two waves were temporally separated as a result of the an 80 -ns delay line in the $532 \mathrm{~nm}$ laser's optical path. The therapy laser was also co-aligned with the two imaging laser pulses, and operated in fire-on-demand mode to photomechanically lyse the CTC upon detection by flow cytography.

In the usual case where there were only RBCs, the $1064 \mathrm{~nm}$ and $532 \mathrm{~nm}$ laser pulses excited both a weak acoustic wave, due to RBCs' weak optical absorption in the NIR, and a strong wave, due to strong absorption of the green laser pulse. When flow cytography scanned a CTC, the early $1064 \mathrm{~nm}$ laser pulse also excited a strong PA pulse, because of the strong absorption of melanosomes. In this event, the late PA signal was contributed by both CTC and RBC absorption of the green laser pulse. All PA signals were concurrently analyzed in the CTC detector, which provided real-time CTC detection and therapy laser triggering. The detector consisted of a time switch and a fast comparator. When PA signals were transmitted into the time switch, it also received a time-gate signal that coincided only with the earlier $1064 \mathrm{~nm}$ laser pulse induced PA signal. Therefore, only the earlier PA signal, which was CTC-specific, was transmitted to the comparator. The comparator compared the CTC-specific PA signal against an optimized pre-set threshold level, and produced a trigger signal to the therapy laser each time a CTC appeared, to lethally irradiate it on the spot. 

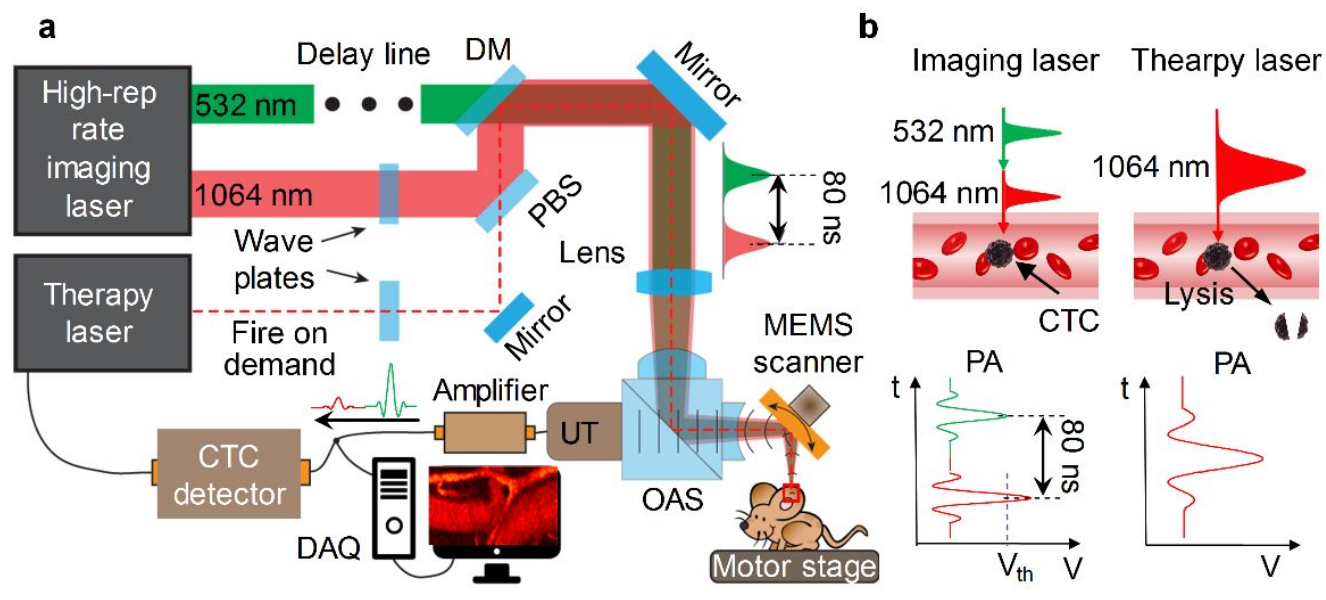

Figure 1. Dual-wavelength PA flow cytography combined with laser therapy. (a) Schematic of the experimental system. DM, dichroic mirror; MEMS, micro-electro-mechanical-system scanner; OAS, opticalacoustic splitter; PBS, polarizing beamsplitter; UT, ultrasonic transducer; DAQ, data acquisition. The $1064 \mathrm{~nm}$ and $532 \mathrm{~nm}$ imaging lasers are employed to image CTCs and vasculature, respectively, and the therapy laser is set on fire-on-demand mode. (b) Scheme of real-time detection and laser killing of CTCs. The CTC detector compares the earlier $1064 \mathrm{~nm}$ laser-induced CTC-specific PA signal against an optimized threshold level (purple dashed line) above the blood signal and noise, and thus can reliably distinguish CTCs and trigger the therapy laser to photomechanically kill the CTC.

\section{RESULTS}

We first used flow cytography to image CTCs with the therapy laser turned off. To simulate melanoma CTCs, a strain of mouse melanoma cell, B16F10, was first cultured and administrated into the mouse's blood circulation through venous or arterial injection. A portion of these cells survived in circulation as CTCs. A region in the mouse ear was imaged by flow cytography at a $\sim 10 \mathrm{~Hz}$ volumetric (3D) rate. Figure 2 shows a single CTC travelling in a vein, and Figure 3 shows snapshots of a $\sim 25-\mu \mathrm{m}$ CTC cluster travelling in an artery. As shown in Figure 2, the CTC is well imaged by $1064 \mathrm{~nm}$ laser-induced PA signals with a high contrast-to-noise ratio, appearing as a bright dot in a dark background. The $532 \mathrm{~nm}$ laser-induced PA image shows that the CTC was travelling inside a vein. A CTC cluster flowing in an artery was also imaged in vivo in a carotid artery cell injection experiment (Figure 3). No return of this CTC cluster was observed up to $30 \mathrm{~min}$ later, which indicated that it might have become clogged at a vascular branching point, seeding a secondary tumor.
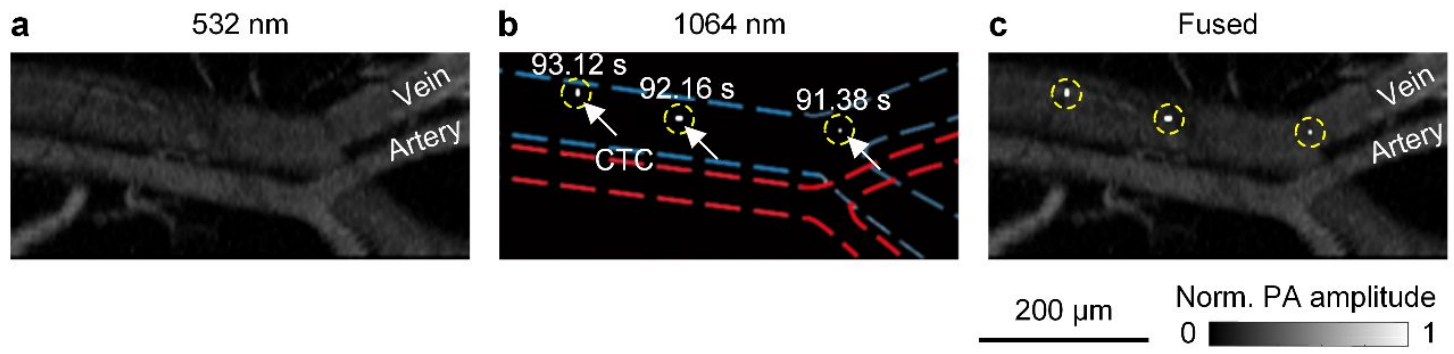

Figure 2. Snapshots showing a single CTC travelling in vasculature. Flow cytography images (a) $532 \mathrm{~nm}$ laser-induced, (b) $1064 \mathrm{~nm}$ laser-induced, and (c) fused flow. In the $1064 \mathrm{~nm}$ laser-induced image, the white arrow and yellow square indicate the detected CTC, and the red and blue dashed lines delineate the artery and vein boundaries, respectively. This recording spans about $\sim 1.8 \mathrm{~s}$, showing a single CTC traveling in the vein. The times labeled in (b) are relative to the injection of the B16F10 cells. 

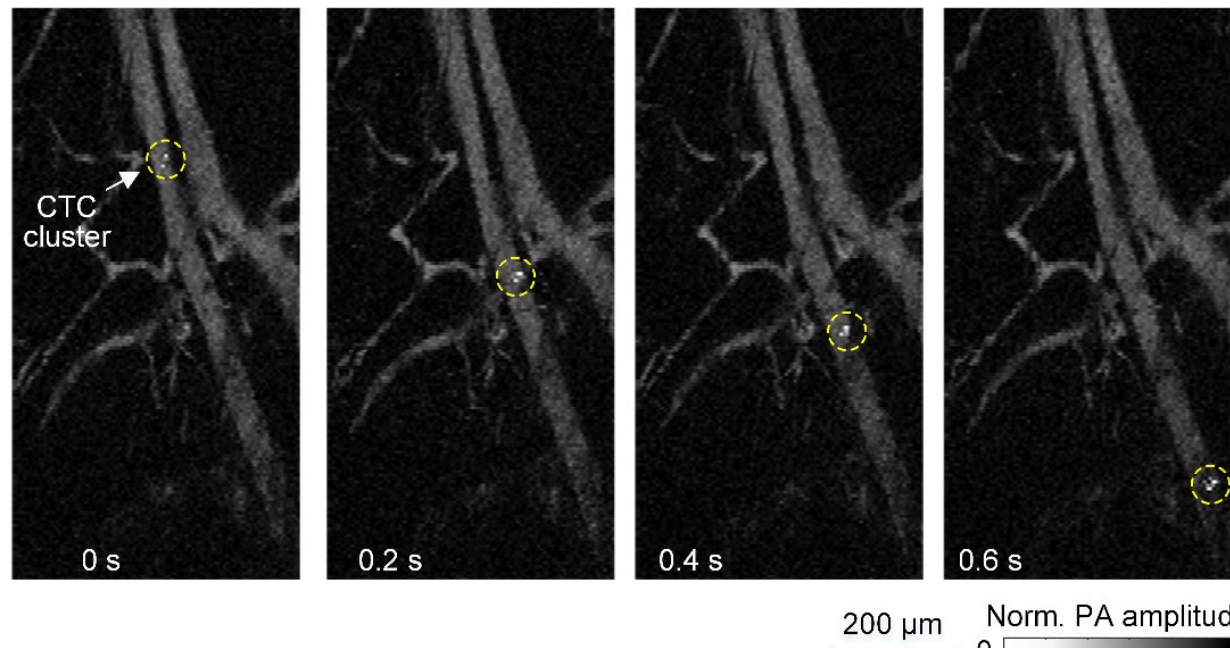

Norm. PA amplitude

Figure 3. Snapshots showing a CTC cluster travelling in an artery. Each image was produced by fusing the $1064 \mathrm{~nm}$ and $532 \mathrm{~nm}$ laser-induced PA flow cytography images. The times labeled at the bottom left of each image are relative to the first image.

We investigated the radiant fluence necessary to kill a CTC by irradiating cultured B16F10 melanoma cells in vitro with a single-shot $1064 \mathrm{~nm}$ pulse from our therapy laser. We found that $8.8 \mathrm{~J} / \mathrm{cm}^{2}$ fluence was sufficient to guarantee cell death. We also found that a $30 \mathrm{~J} / \mathrm{cm}^{2}$ fluence irradiation did not cause hemolysis or any RBC morphology change visible under microscope observation.

We also performed in vivo CTC photothermolysis experiments with on-the-fly CTC detection and destruction. As shown in Figure 4, the CTC signal induced by the $1064 \mathrm{~nm}$ laser pulse typically had a $\sim 20$ contrast-to-noise ratio, which enabled the CTC detector to easily threshold it out and to trigger the therapy laser using hardware. Within 10 $\mu \mathrm{s}$, an energetic pulse was fired from the therapy laser, which followed the optical path of the imaging laser pulses and focused onto the CTC location with a $50 \mu \mathrm{m}$ focal diameter and a $25 \mathrm{~J} / \mathrm{cm}^{2}$ fluence, above the lethal level. This lethal irradiation on the CTC produced a PA signal with a much greater amplitude, $>14$ times higher than the usual imaging signals (Figure 4c). The peak location of this signal indicated that the CTC was within its focal spot. Therapy laser-induced PA signals were also studied in another experiment. As shown in Figure 4d, the amplitude of the signals induced by the CTC-detector-triggered therapy laser pulse, after detection of a CTC occurrence, was much greater than that induced by therapy laser pulses fired onto the same blood vessel using a manual trigger. Therefore, we concluded that this PA signal mainly originated from the CTC absorption, and that this CTC was lysed by the lethal therapy laser irradiation. In total, four events of real-time CTC detection and expected photothermal killing were recorded in this experiment. Flow cytography confirmed no disturbance to the blood flow during 30 min of observation after this experiment, as did visual observation of the imaging area. 
a

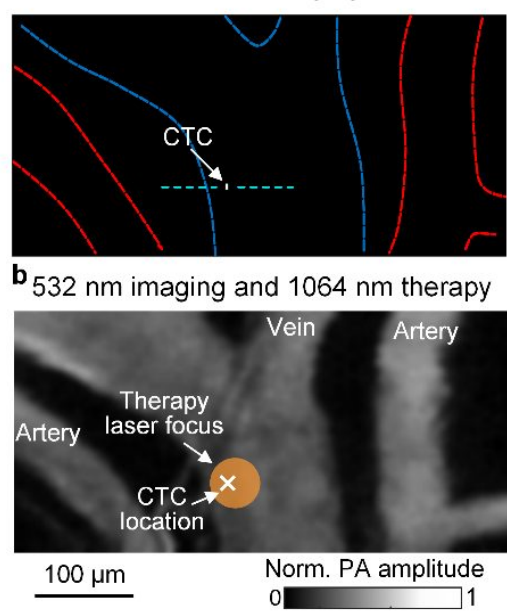

c

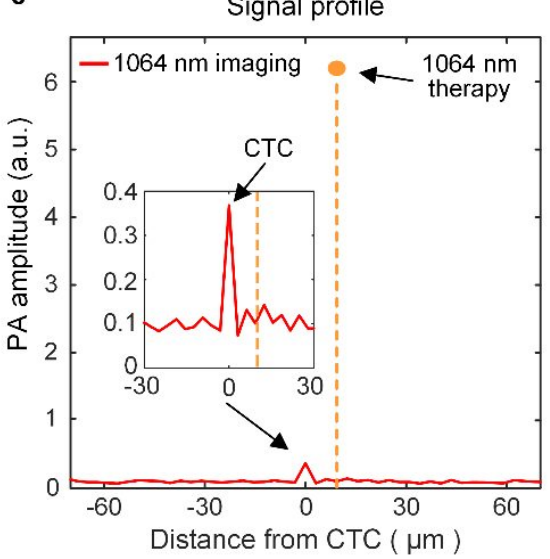

d

Therapy laser-induced PA signals

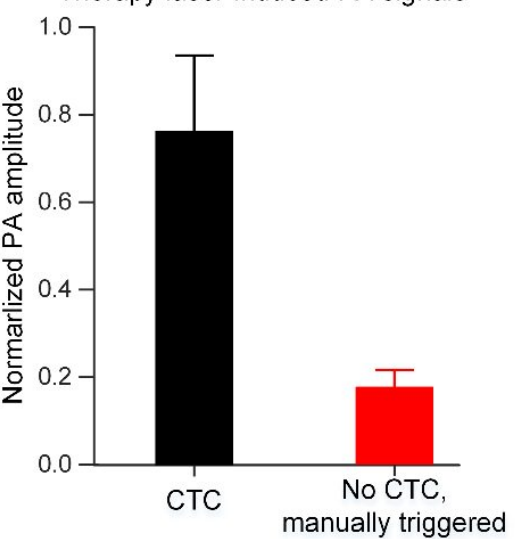

Figure 4. In vivo real-time detection and expected photothermal lysis of a CTC. The CTC signal in the 1064 nm laser-induced flow cytography image (a) has a $\sim 25$ contrast-to-noise ratio (c), which is thresholded out by the CTC detector to trigger the therapy laser. In the $532 \mathrm{~nm}$ laser-induced image (b), the therapy laser-induced PA signal is illustrated by the filled orange circle. (c) PA signal profile from the region across the CTC, indicated by the dashed cyan line in (a). The $x$ axis, parallel with the imaging laser's scanning direction (from left to right), is centered at the CTC location. The peak location of the therapy laser-induced PA signal (dashed orange line) is only $\sim 10 \mu \mathrm{m}$ away from the detected CTC location, indicating that the CTC position (white cross in (b)) was within the therapy laser's focal spot (orange circle in (b)). (d) Study of therapy laser-induced PA signals. Black bar: PA signals induced by the therapy laser when it was triggered by the CTC detector after detecting a CTC. Red bar: PA signals induced by the therapy laser when it was manually triggered and fired onto the same blood vessel when it did not contain a CTC. Data are averaged over ten measurements; error bars: standard deviations.

\section{DISCUSSION}

We report dual-wavelength PA flow cytography combined with on-the-fly therapy laser administration for both in vivo label-free imaging and on-the-spot selective photothermolysis of melanoma CTCs. The CTC count is an important indicator of cancer severity and the efficacy of tumor therapies, making the reliable detection of CTCs clinically significant. Our detection method relies on the optical absorption property of CTCs, instead of more elusive biological properties. This technique can be translated clinically for non-invasive in vivo CTC assays for evaluating tumor metastasis risks. In addition, our technology can also be used for studying the biological processes of tumor metastasis through CTCs, such as imaging CTC dynamics.

Unlike therapeutics that use metastasis-specific features to guide the translocation of administrated drugs, our method employs a more selective "identify-then-locally-administer" procedure. Because of the $\sim 500$-nm thermal diffusion range, the therapy laser pulse is highly localized on the melanosomes within CTCs, so other cellular components, as well as adjacent RBCs, are not exposed to excessive radiant energy. Drug resistance is also not an issue for our method because the destruction mechanism is entirely physical.

In summary, using endogenous absorption contrast, our system successfully imaged rare single CTCs and CTC clusters in vivo and performed real-time pinpoint photothermolysis of single CTCs. This technology works in a convenient reflection mode, and is easily translatable to clinics. It can serve as a bedside detection and therapy device for cancer patients, which can reduce metastasis risks by early detection and destruction of CTCs. The technology can also be applied for studying the biological process of CTC-related tumor metastasis.

\section{REFERENCES}

[1]D. L. Cummins, J. M. Cummins, H. Pantle, M. A. Silverman, A. L. Leonard, and A. Chanmugam, "Cutaneous malignant melanoma," Mayo Clinic proceedings, 81(4), 500-507 (2006). 
[2]P. S. Steeg, "Tumor metastasis: mechanistic insights and clinical challenges," Nature medicine, 12(8), 895-904 (2006).

[3] V. Plaks, C. D. Koopman, and Z. Werb, "Circulating tumor cells," Science, 341(6151), 1186-1188 (2013).

[4] L. Khoja, P. Lorigan, C. Dive, U. Keilholz, and A. Fusi, "Circulating tumor cells as tumor biomarkers in melanoma: detection methods and clinical relevance," Annals of Oncology, mdu207 (2014).

[5]S. A. Joosse, and K. Pantel, "Biologic challenges in the detection of circulating tumor cells," Cancer research, 73(1), 8-11 (2013).

[6] S. L. Jacques, "Optical properties of biological tissues: a review," Physics in medicine and biology, 58(11), R37 (2013).

[7] L. V. Wang, "Tutorial on photoacoustic microscopy and computed tomography," Selected Topics in Quantum Electronics, IEEE Journal of, 14(1), 171-179 (2008).

[8] Y. Zhou, G. Li, L. Zhu, C. Li, L. A. Cornelius, and L. V. Wang, "Handheld photoacoustic probe to detect both melanoma depth and volume at high speed in vivo," Journal of biophotonics, 8(11-12), 961-967 (2015).

[9] Y. He, L. Wang, J. Shi, J. Yao, L. Li, R. Zhang, C.-H. Huang, J. Zou, and L. V. Wang, "In vivo labelfree photoacoustic flow cytography and on-the-spot laser killing of single circulating melanoma cells," Scientific Reports, 6, 39616 (2016).

[10] A. Schroeder, D. A. Heller, M. M. Winslow, J. E. Dahlman, G. W. Pratt, R. Langer, T. Jacks, and D. G. Anderson, "Treating metastatic cancer with nanotechnology," Nature Reviews Cancer, 12(1), 39-50 (2012).

[11] E. I. Galanzha, E. V. Shashkov, P. M. Spring, J. Y. Suen, and V. P. Zharov, "In vivo, noninvasive, label-free detection and eradication of circulating metastatic melanoma cells using two-color photoacoustic flow cytometry with a diode laser," Cancer research, 69(20), 7926-7934 (2009).

[12] J. L. Thomas, T. S. Lister, S. L. Royston, and P. A. Wright, "Adverse effects following Qswitched ruby laser treatment of pigmented lesions," Journal of Cosmetic and Laser Therapy, 12(2), 101-105 (2010).

[13] R. R. Anderson, and J. A. Parrish, "Selective photothermolysis: precise microsurgery by selective absorption of pulsed radiation," Science, 220(4596), 524-527 (1983). 